\title{
NOS1 upregulates ABCG2 expression contributing to DDP chemoresistance in ovarian cancer cells
}

\author{
XIAOXUAN LI $^{1 *}$, ZHIWEI ZOU ${ }^{2 *}$, JIAO TANG $^{1}$, YOUHONG ZHENG ${ }^{1}$, \\ YU LIU ${ }^{1}$, YAN LUO ${ }^{1}$, QIUZHEN LIU² ${ }^{2}$ and YIFENG WANG ${ }^{1}$ \\ ${ }^{1}$ Department of Obstetrics and Gynecology, Zhujiang Hospital, Southern Medical University, Guangzhou, \\ Guangdong 510280; ${ }^{2}$ Cancer Research Institute, School of Basic Medical Sciences, \\ Southern Medical University, Guangzhou, Guangdong 510515, P.R. China
}

Received January 15, 2016; Accepted November 27, 2018

DOI: $10.3892 / \mathrm{ol} .2018 .9787$

\begin{abstract}
Nitric oxide synthase 1 (NOS1) has been reported to promote various cancer processes including chemoresistance. However, the role of NOS1 in chemoresistance has remained unclear. ATP-binding cassette, subfamily G, member 2 (ABCG2) has been identified as a molecular cause of multidrug resistance in a number of cancer types, including ovarian cancer. The present study observed that in ovarian cancer cells, the expression of ABCG2 was significantly upregulated in response to cis-diamminedichloroplatinum (cisplatin/DDP) treatment, in addition the expression of NOS1 exhibited an increasing trend. Additionally, the levels of NOS1 and ABCG2 in chemoresistant ovarian cancer profiles in Gene Expression Omnibus datasets (GSE26712 and GSE51373) were higher than in chemosensitive profiles. Furthermore, overexpression of NOS1 could upregulate ABCG2 expression, and expression of ABCG2 was inhibited by NOS1 selective inhibitor (N-PLA). In assays of cell survival, NOS1 appeared to increase the potential for DDP resistance, and this effect was reversed by addition of ABCG2 inhibitor (verapamil). The present study indicated that NOS1-induced chemoresistance was partly mediated by the upregulation of ABCG 2 expression. This
\end{abstract}

Correspondence to: Professor Yifeng Wang, Department of Obstetrics and Gynecology, Zhujiang Hospital, Southern Medical University, 283 Gongye Avenue Central, Guangzhou, Guangdong 510280, P.R. China

E-mail: yifengw1963@sina.com

Professor Qiuzhen Liu, Cancer Research Institute, School of Basic Medical Sciences, Southern Medical University, 1838 Guangzhou Avenue North, Guangzhou, Guangdong 510515, P.R. China

E-mail: liuqiuzhen@126.com

${ }^{*}$ Contributed equally

Key words: nitric oxide synthase 1, ATP-binding cassette, subfamily G, member 2, ovarian cancer, chemoresistance, cis-diamminedichloroplatinum result suggests a link between the expression of NOS1 and the ABCG2-associated chemoresistance in ovarian cancer.

\section{Introduction}

Epithelial ovarian cancer (EOC) is among the most common gynecological malignancies. Despite substantial improvements in surgery and chemotherapy, the survival rate of patients with EOC remains low, since the majority of patients with ovarian cancer relapse and/or become drug-resistant following a period of chemotherapy (1). Thus, identifying the potential biomarkers of chemoresistance is of great significance for EOC.

Nitric oxide (NO) is a lipophilic, highly diffusible and short-lived physiological messenger (2). Endogenous NO may be produced by different isoforms of NO synthase (NOS), including NOS1, 2 and 3, during arginine metabolism. Among the three types of NOS; NOS1 and 3, are constitutively expressed in cells and thus termed as cNOS, may produce a low level of $\mathrm{NO}$ transiently in a $\mathrm{Ca}^{2+}$-dependent manner (3). Meanwhile, NOS2, which is induced by inflammatory factors and cytokines in a $\mathrm{Ca}^{2+}$-independent manner, generates high and sustained concentrations of NO (4). At high concentrations (>500 $\mathrm{nM})$, NO induces an inflammatory reaction, apoptosis and other process that inhibit tumor biological functions, whereas NO at lower, more physiological levels $(<100 \mathrm{nM})$ typically exhibits the opposite effects, promoting tumor behaviors including metastasis, angiogenesis and chemoresistance $(5,6)$. Previously, chemoresistant phenotypes of colon cancer, head and neck cancer and human breast carcinoma cells have been reported to be associated with different NOS isoforms $(5,7,8)$. NOS2-derived NO may induce apoptosis in cancer cells, while the lower concentration of NO typically generated by NOS1 may inhibit apoptosis and promote chemoresistance (9). However, the mechanism underlying NOS1 associated chemoresistance is unclear.

ATP-binding cassette, subfamily G, member 2 (ABCG2), also known as breast cancer resistance protein, was originally cloned from multidrug-resistant breast cancer cells, and its upregulation has been linked to chemoresistance in various cancer cells, including ovarian cancer (10). ABCG2 extrudes xenobiotics and certain drugs from cells, thereby mediating drug resistance and affecting the pharmacological behavior 
of many compounds (11). Additionally, ABCG2 has been revealed to be a transporter of glutathione, which is vital for the maintenance of cellular redox balance (12). It was also been documented that human ABCG2 could limit the uptake of pheophorbide-A and many porphyrin derivatives, both of which can produce singlet oxygen, which causes DNA fragmentation and caspase-3 dependent apoptosis (12). Therefore, ABCG2 may play an important role in chemotherapy resistance through its redox related function.

It has been reported that NO endogenously produced by NOS enzymes promotes the transcriptional activity of antioxidant-related transcription factors, including hypoxia-inducible factor (HIF)-1 $\alpha$ and nuclear factor erythroid 2-related factor 2 (Nrf2); these then induce transcription of antioxidant factors including superoxide dismutase and thioredoxin reductase 1 , thereby increasing the ability of cancer cells to resist chemotherapy-induced reactive oxygen species stress, and thus decreasing sensitivity to chemotherapy-induced apoptosis (13-16). Cis-diamminedichloroplatinum (cisplatin/DDP), a chemotherapy drug, destroyed tumor cells by binding to DNA strands, interfering with DNA replication. DDP has been one of first lines of defense against tumors, especially those of ovary, lung and testes (17). However to date, it has remained to be investigated whether NOS1/N-related chemotherapy resistance is associated with the multidrug resistance-associated gene ABCG2 in the regulation of redox balance.

In the present study, the plausible mechanisms of NOS1 contribution to DDP resistance via ABCG2 in EOC were investigated. In order to detect the expression changes of NOS1 and ABCG2 prior to and following DDP incubation, western blot analysis was performed to detect the protein expression level. Additionally, the levels of NOS1 and ABCG2 in chemoresistant and chemosensitive ovarian cancer profiles were detected by GEO database analysis. In addition, protein levels of ABCG2 were detected by western blot and immunohistochemistry (IHC) staining, in order to investigate the impact of NOS1 on ABCG2, MTT and flow cytometry analysis were used to detect cell viability and apoptosis. The present results may provide a basis for chemotherapeutic improvement via NOS1 inhibition.

\section{Materials and methods}

Reagents and plasmids. The following chemicals and plasmids were used: 3-(4,5-dimethylthiazol-2-yl)-2,5-diphenyltetrazolium bromide (MTT), dimethyl sulfoxide (DMSO), ABCG2 inhibitor verapamil hydrochloride $(20 \mu \mathrm{M}$; used in the MTT assay), specific NOS1 inhibitor $\mathrm{N}^{\omega}$-propyl-L-arginine hydrochloride (N-PLA), specific NOS2 inhibitor 1400W dihydrochloride and general NOS inhibitor $\mathrm{N}^{\omega}$-nitro-L-arginine methyl ester hydrochloride (L-NAME) (all Sigma-Aldrich; Merck KGaA, Darmstadt, Germany); DETA-NONOate was purchased from Cayman Chemical Company (Ann Arbor, MI, USA); FastQuant RT kit and SYBR-Green PCR kit (Takara Bio, Inc., Otsu, Japan); rabbit monoclonal anti-NOS1 antibody (ab76067) and mouse monoclonal anti-ABCG2 antibody (ab3380; Abcam, Cambridge, MA, USA); mouse monoclonal anti-GAPDH antibody (ZSGB-BIO, Beijing, China); a fluorescein isothiocyanate-Annexin $\mathrm{V}$ apoptosis detection kit (BD Biosciences, San Jose, CA, USA); TRIzol reagent (Invitrogen; Thermo Fisher Scientific, Inc., Waltham, MA, USA); lentiviral plasmid GV375-NOS1, negative control GV375-NC, enhanced infection solution and polybrene (Shanghai GeneChem Co., Ltd., Shanghai, China); small interfering (si)RNAs for NOS1 and negative control siRNA (Guangzhou Ribobio Co., Ltd., Guangzhou, China); Lipofectamine 2000 reagent (Thermo Fisher Scientific, Inc.); and an IHC universal SP test kit (ZSGB-BIO).

GEO database assays analysis. Gene expression profiles from the Gene Expression Omnibus database (www.ncbi.nlm.nih. gov/geo; GSE26712 and GSE51373) were downloaded. The data in GSE26712 included 185 advanced ovarian cancer cases, and in GSE51373 were 28 high-grade serous ovarian cancer cases. Serous ovarian carcinoma is divided into high-grade serous (HGSOC) and low-grade serous ovarian carcinoma (LGSOC). The two-tier system based on nuclear atypia was introduced in 2004 by the University of Texas M.D. Anderson Cancer Center (MDACC) (18). Despite large amount of gene expression data in the assays, we only analyzed the expression data of ABCG2 and NOS1. The correlation of expression of NOS1 and ABCG2 was analyzed by Pearson correlation coefficient analysis. In addition, the expression differences of NOS1 and ABCG2 were detected in DDP-sensitive and DDP-resistant patients.

Cell culture and transfection. The human ovarian cancer lines OVCAR-3 and SKOV-3, purchased from the Chinese Academy of Sciences (Shanghai, China), were grown in Dulbecco's modified Eagle's medium (Gibco; Thermo Fisher Scientific, Inc.) supplemented with $10 \%$ fetal bovine serum (BI, Salt Lake City, UT, USA; www.bioind.com/worldwide/), 100 U/ml penicillin and $100 \mu \mathrm{g} / \mathrm{ml}$ streptomycin, and incubated at $37^{\circ} \mathrm{C}$ in a humidified chamber supplemented with $5 \% \mathrm{CO}_{2}$.

The lentiviral plasmid GV375-NOS1 and negative control GV375-NC were individually transfected into OVCAR-3 cells, and the enhanced infection solution and polybrene were used to concentrate the lentiviral supernatant. Briefly, $3 \times 10^{4}$ OVCAR-3 cells were plated in 24-well culture plates. When the confluence reached $20-30 \%$, lentiviral supernatant was added to the wells ( $5 \mu \mathrm{l} /$ well, $1 \times 10^{8}$ transducing units $/ \mathrm{ml}$ ). Puromycin $(5 \mu \mathrm{g} / \mathrm{ml})$ was used to select for positive clones. The transfected OVCAR-3 cells (hereafter termed GV375-NOS1 ov-3 and GV375-NC ov-3) were used in further experiments.

The sequence of the NOS1 siRNA was: Forward, 5'-GGU CUAUCCAAUGUCCACAdTdT-3' and reverse, 3'-dTdTCC AGAUAGGUUACAGGUGU-5'. The target sequence was: GGTCTATCCAATGTCCACA. The siRNAs for NOS1 and the negative control (si-NC) were designed and synthesized by Guangzhou Ribobio Co., Ltd. The NOS1-specific and negative control siRNAs (100 nM, Transfection efficiency: >80\%) were transfected into SKOV-3 cells using Lipofectamine 2000 reagent according to the manufacturer's protocol. Cells were then incubated at $37^{\circ} \mathrm{C}$ for $48-72 \mathrm{~h}$ and collected to assess transfection efficiency by western blotting and real time PCR, and were then used in further experiments.

Flow cytometry analysis. Cultured cells were trypsinized and resuspended in phosphate-buffered saline (PBS). To examine whether NOS is involved in promoting platinum-based 
chemotherapy resistance, apoptosis induction was detected by flow cytometric analysis using a FACS Aria II (BD Biosciences). For this, a fluorescein isothiocyanate-Annexin $\mathrm{V}$ apoptosis detection kit was used according to the manufacturer's instructions. Cells were incubated with $2 \mu \mathrm{M}$ DDP only or $2 \mu \mathrm{M}$ DDP plus either $1 \mathrm{mM}$ L-NAME, $100 \mu \mathrm{M}$ N-PLA, $100 \mu \mathrm{M} 1400 \mathrm{~W}$ or $20 \mu \mathrm{M}$ DETA-NONOate at $37^{\circ} \mathrm{C}$ for $48 \mathrm{~h}$ without changing the medium. The ratios of apoptotic cells in the treated and untreated groups were analyzed with FlowJo software (version 7.6.1; Tree Star, Inc., Ashland, OR, USA).

Assessment of cell viability. OVCAR-3 and SKOV-3 cell viabilities were determined by MTT assay following transfection or treatment with different drugs $(2 \mu \mathrm{M}$ DDP, $20 \mu \mathrm{M}$ verapamil, $1 \mathrm{mM}$ L-NAME, $100 \mu \mathrm{M}$ N-PLA, $100 \mu \mathrm{M}$ $1400 \mathrm{~W}$ or $20 \mu \mathrm{M}$ DETA-NONOate) for $48 \mathrm{~h}$. Briefly, cells were seeded in 96-well tissue culture plates $(100 \mu \mathrm{l} /$ well $)$ at a density of $5 \times 10^{3}$ cells/well. After the cell monolayer reached $50-60 \%$ confluence, the culture medium of each well was refreshed with $100 \mu \mathrm{l}$ medium combined with drugs as described above. After $48 \mathrm{~h}$, the cells were incubated with MTT solution ( $2 \mathrm{mg} / \mathrm{ml}$ ) for $4 \mathrm{~h}$. Subsequently, the MTT solution was removed and DMSO (150 $\mu \mathrm{l} /$ well) was added. A Multiskan Spectrum spectrophotometer (Thermo Fisher Scientific, Inc.) was used for measuring absorbance at $490 \mathrm{~nm}$. The half maximal inhibitory concentration (IC50) was calculated using the following equation: IC $50=($ mean OD of specific treatment group)/(mean OD of negative control group)\%.

RNA extraction and real-time PCR. Total RNA from L-NAME-treated and untreated OVCAR-3 cells was extracted with TRIzol Reagent following the manufacturer's protocol. First strand cDNA synthesis and amplification were performed using a FastQuant RT kit. The cDNA was used to amplify ABCG2 and endogenous control GAPDH via PCR. SYBR-Green was used for quantitative PCR. The PCR cycles were as follows: $95^{\circ} \mathrm{C}$ for $15 \mathrm{~min}$, followed by 40 cycles of $95^{\circ} \mathrm{C}$ for $10 \mathrm{sec}$ and $60^{\circ} \mathrm{C}$ for $32 \mathrm{sec}$, performed in a Mx3005 Sequence Detection system (Stratagene, La Jolla, CA, USA). The comparative $C_{q}\left(\Delta \Delta C_{q}\right)$ method (19) was used to determine the expression fold change. The primer sequences used were as follows: For GAPDH forward, 5'-GAAGGTGAAGGTCGG AGTC-3' and reverse, 5'-GAAGATGGTGATGGGATTTC-3'; and for ABCG2, forward, 5'-ACGAACGGATTAACAGGG TCA-3' and reverse, 5'-CTCCAGACACACCACGGAT-3'.

Western blot analysis. OVCAR-3 cells following transfection or treatment with different drugs (DDP, L-NAME, N-PLA or $1400 \mathrm{~W}$ ) for $48 \mathrm{~h}$ and negative control cells were lysed in a radioimmunoprecipitation assay lysis buffer (Thermo Fisher Scientific, Inc.) containing $1 \mathrm{mM}$ phenylmethylsulfonyl fluoride. The proteins were separated by $10 \%$ SDS denaturing polyacrylamide gel and then transferred onto polyvinylidene difluoride membranes. The membranes were blocked in $5 \%$ bovine serum albumin (BI, Salt Lake City, UT, USA) at room temperature for $2 \mathrm{~h}$, and blotted with the mouse antihuman antibody against ABCG2 and the rabbit anti-human antibody against NOS1 at their respective recommended dilution $(1: 1,000)$ overnight at $4^{\circ} \mathrm{C}$, and then incubated with the appropriate secondary antibody (FD0128; FD0142; 1:5,000; Fude
Biological Technology Co. Ltd. China) at room temperature for $1 \mathrm{~h}$. Following a wash with tris-buffered saline with Tween-20, visualization of the secondary antibody was performed using a chemiluminescence detection assay (ChemiDoc XRS+ Imaging System; Bio-Rad Laboratories, Inc., Hercules, CA, USA) according to the manufacturer's protocol. Image Lab software (version 3.0.1 Bio-Rad Laboratories, Inc.) was used to quantify band intensities. GAPDH $(1: 1,000)$ was used as a loading control.

Animal experiments. Animal experiments were approved by the Ethical Committee of Southern Medical University (Guangzhou, China). A total of 12 mice were randomly assigned to two groups ( $n=6 /$ group) of BALB/c-nu female mice (4-6 weeks old; $18-20 \mathrm{~g}$ ) were housed in specific pathogen-free facilities under 12-h light/dark cycles. The feeding environment temperature was $18-29^{\circ} \mathrm{C}$ and the humidity was $40-70 \%$. Animals' foods were sterile and highly nutritional and water was clean filtered, access to food and water was ad libitum. A total of 5x106 GV375-NOS1 ov-3 or GV375-NC ov-3 cells were pelleted (450 x g for $5 \mathrm{~min}$ at room temperature), resuspended in $100 \mathrm{ml}$ Matrigel (BD Biosciences) and injected into the right flank of nude mice. The tumor volumes were calculated with the formula: Volume $\left(\mathrm{mm}^{3}\right)=$ width $\mathrm{x}$ width $\mathrm{x}$ length $\mathrm{x} 0.5$. Mice were sacrificed when tumors reached $50 \mathrm{~mm}^{3}$, and the tumor tissues were excised and weighed.

IHC staining. The tumor tissues were fixed by $10 \%$ neutral buffered formalin (Wexis, Guangzhou, China) at room temperature for $24 \mathrm{~h}$. The slides ( $5 \mathrm{~mm}$ sections) of xenografted tumors were deparaffinized using xylene and dehydrated using ethanol, then washed in PBS. For antigen unmasking, slides were placed in a container, covered with citrate buffer (pH 6.0) and heated in a steamer for $5 \mathrm{~min}$ at $100^{\circ} \mathrm{C}$. The slides were then washed and blocked with $5 \%$ normal goat serum (BioLab, Beijing, China; http://www.hbzhan. com/st161595/erlist_692382.html) for $30 \mathrm{~min}$, then incubated with primary antibody (anti-ABCG $2,1: 100$ ) overnight at $4^{\circ} \mathrm{C}$, and subsequently stained with horseradish peroxidase-coupled secondary antibodies (FD0128; 1:200; FD Science, Hangzhou, China) at room temperature for $30 \mathrm{~min}$. The slides were then visualized using an upright light microscope (Nikon Corporation, Tokyo, Japan).

Statistical analysis. Statistical analyses were performed in SPSS version 19.0 software for Windows (IBM, Corp., Armonk, NY, USA). All experiments were performed at least in triplicate. The expression levels of NOS1 and ABCG2 were compared between two groups by Student t-tests. Multiple comparisons were performed by a one-way analysis of variance followed by a least significant difference post-hoc test. Pearson's correlation analysis was used to assess the association between the mRNA levels of NOS1 and ABCG2. Data are presented as the mean \pm standard deviation. Significance was determined at $\mathrm{P}<0.05$.

\section{Results}

Expression of NOS1 and ABCG2 is increased following treatment with $D D P$. To determine the association between the expression of NOS1 and ABCG2 and the chemoresistant 

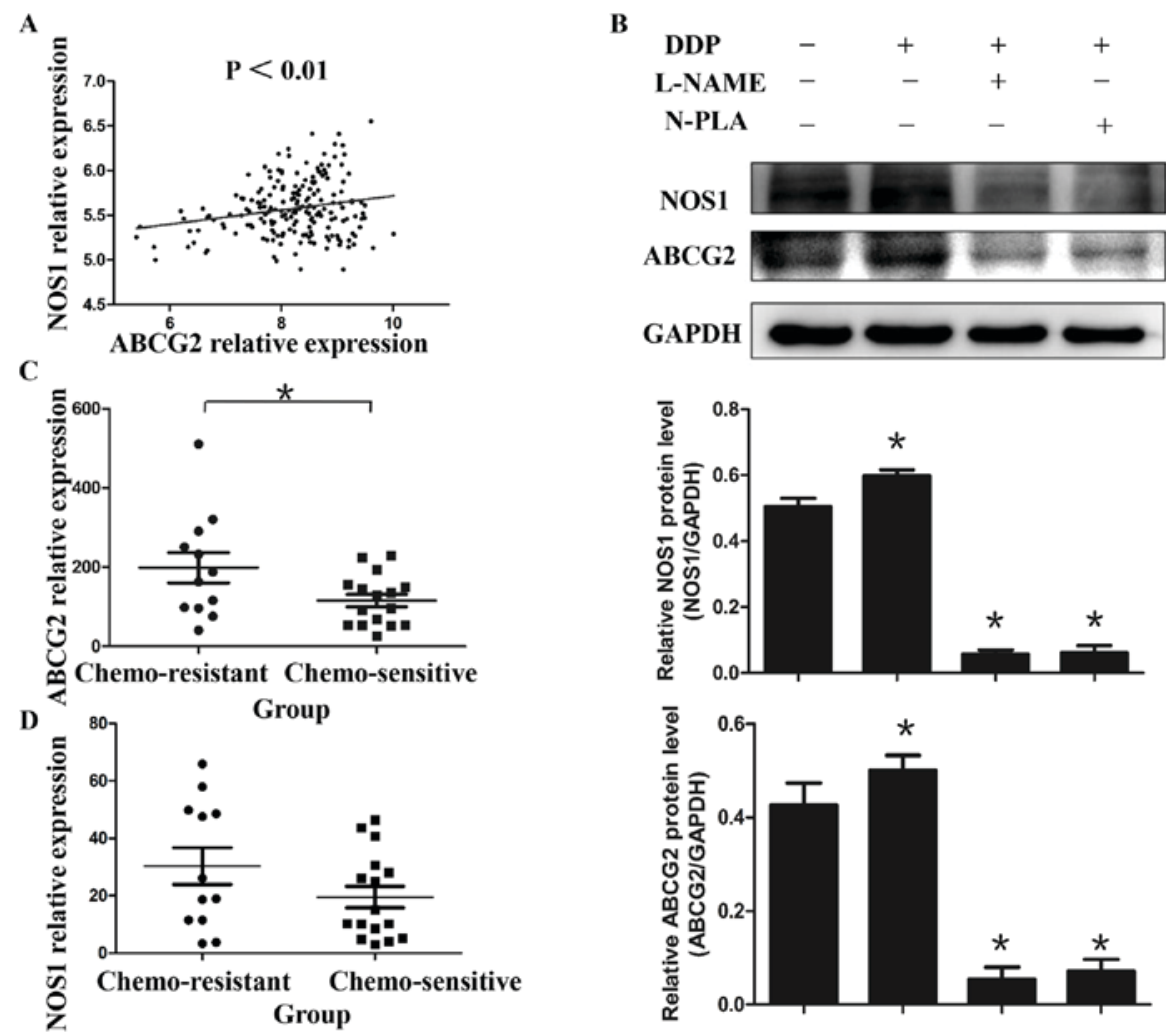

Figure 1. Expression levels of NOS1 and ABCG2 were increased following treatment with DDP. (A) NOS1 expression was significantly positively correlated with ABCG2 expression. "P<0.05. (B) DDP treatment increased NOS1 and ABCG2 protein levels, and L-NAME and N-PLA reversed this effect. ${ }^{*} \mathrm{P}<0.05$. (C and D) mRNA levels of NOS1 and ABCG2 were higher in DDP-resistant than DPP-sensitive ovarian cancer tissues. "P<0.05. NOS1, nitric oxide synthase 1; ABCG2, ATP-binding cassette, subfamily G, member 2; DPP, cisplatin/cis-diamminedichloroplatinum; L-NAME, $\mathrm{N}^{\omega}$-nitro-L-arginine methyl ester hydrochloride; N-PLA, $\mathrm{N}^{\omega}$-propyl-L-arginine hydrochloride.

phenotype in EOC, the mRNA levels of NOS1 and ABCG2 and their potential correlation was investigated in gene expression profiles from the Gene Expression Omnibus database (www. ncbi.nlm.nih.gov/geo; GSE26712 and GSE51373; Fig. 1). It was identified that the mRNA levels of NOS1 and ABCG2 were significantly correlated in EOC (Fig. $1 \mathrm{~A} ; \mathrm{r}=0.2115$; $\mathrm{P}<0.01$ ). Furthermore, the expression of the ABCG2 gene was higher in chemoresistant EOC tissues compared with that in chemosensitive tissues represented in GSE26712 and GSE51373, but no significant difference was observed in the mRNA levels of NOS1 (Fig. 1C and D). This phenomenon was verified in vitro by evaluating alteration of the protein levels of NOS1 and ABCG2 in EOC cell lines following DDP treatment. It was observed that the treatment with DDP $(2 \mu \mathrm{M}, 48 \mathrm{~h})$ increased the protein levels of ABCG2 and NOS1 when assessed by immunoblotting. Notably, this enhanced expression of both genes by DDP treatment could be reduced by the non-selective NOS inhibitor L-NAME or the NOS1 selective inhibitor N-PLA (Fig. 1B; P<0.05), suggesting that NOS1 may regulate the expression of ABCG2.

NOS1 upregulates the expression of $A B C G 2$. It has been demonstrated that NOS1 may promote the transcription of numerous genes by activating transcription factors $(15,20)$. The change of ABCG2 expression in OVCAR-3 cells was analyzed in response to functional modulation of NOS by L-NAME treatment. As depicted in Fig. 2A, the mRNA and protein levels of ABCG2 were decreased following L-NAME treatment detected by real-time PCR $(0.36$-fold; $\mathrm{P}<0.05)$ and western blot analysis $(\mathrm{t}=44.43, \mathrm{P}<0.05)$. The experiments were repeated 3 times. Subsequently, the different contributions of NOS isoforms to ABCG2 regulation was analyzed using NOS selective inhibitors. The expression of ABCG2 was downregulated by N-PLA in comparison with that in control cells (Fig. 2B, P<0.05). By contrast, L-NAME and the NOS2 specific inhibitor 1400W did not induce a change in ABCG2 expression ( $\mathrm{P}>0.05)$. The impact of NOS1 gene overexpression on the expression of ABCG2 was next investigated by comparing the expression levels of ABCG2 in OVCAR-3 cells constitutively overexpressing NOS1 (GV375-NOS1 ov-3) and the negative controls (GV375-NC ov-3). It was observed that the overexpression of NOS1 upregulated ABCG2 expression when compared with in the negative control group (Fig. 2C; NOS1:t=5.006, $\mathrm{P}<0.05 ; \mathrm{ABCG} 2: \mathrm{t}=17.89, \mathrm{P}<0.05)$. The experiments were repeated 3 times.

Furthermore, GV375-NOS1 ov-3 and GV375-NC ov-3 cells were injected into the backs of nude mice. The expression of ABCG2 was detected in the xenograft cell model by IHC staining, and it was found that the overexpression of NOS1 increased the expression of ABCG2 in the resulting tumor tissues of nude mice (Fig. 2D). These results suggested that NOS1 expressed by ovarian cancer cells could increase the expression of $\mathrm{ABCG} 2$ in vitro and in vivo.

NOS1 contributes to DDP resistance in ovarian cancer cell lines. Two ovarian cancer cell lines with differing DDP resistance, OVCAR-3 and SKOV-3, were examined by MTT 
A
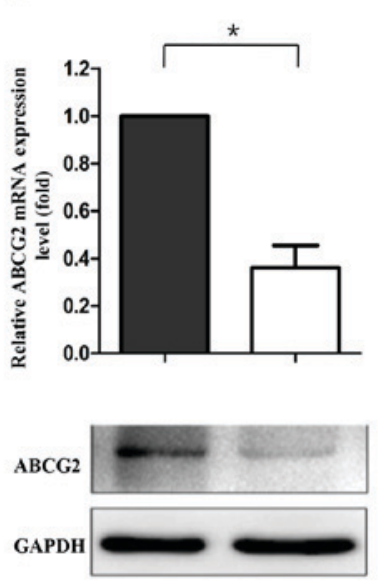
CON L-NAME

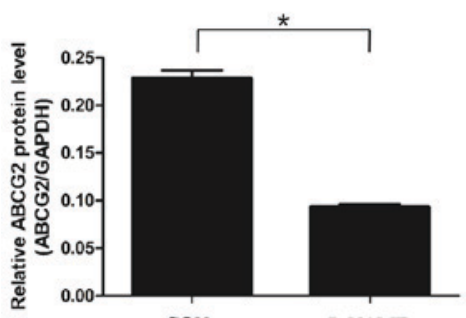

CON

C

D
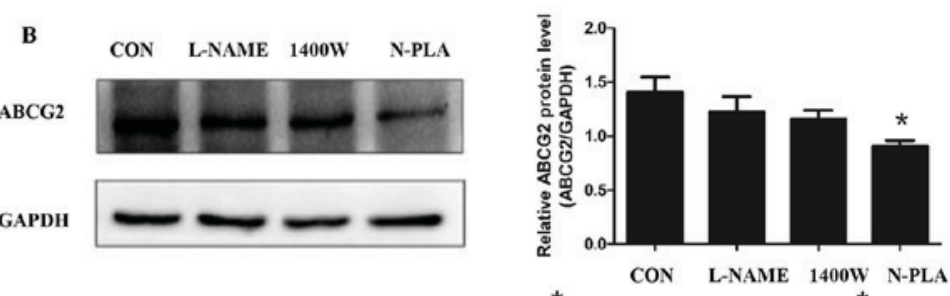
Nos1 ABCG2 GAPDH
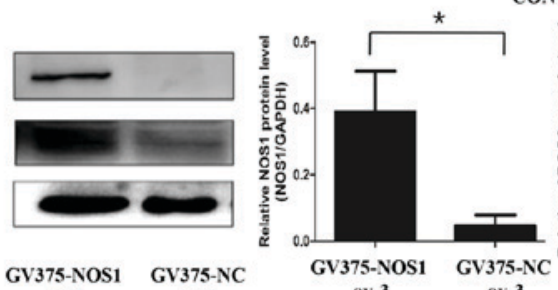
ov-3

ov-3
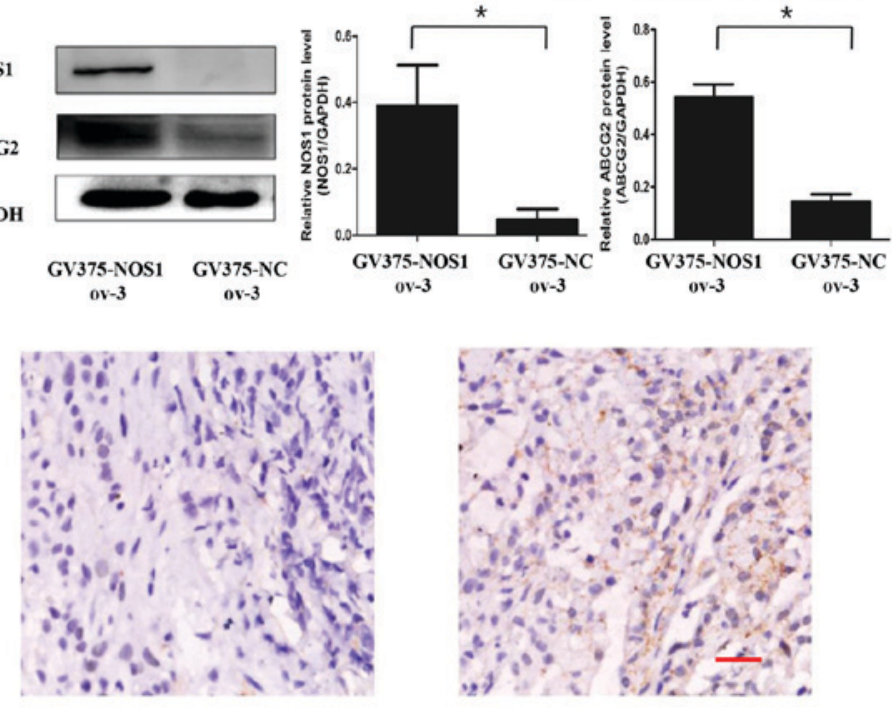

GV375-NC Group

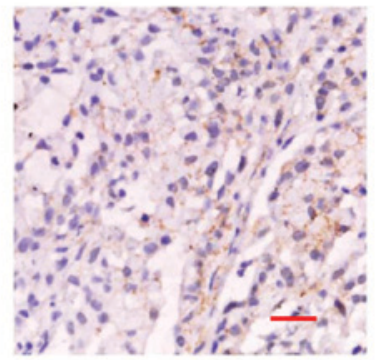

GV375-NOS1 Group

Figure 2. NOS1 upregulated the expression of ABCG2. (A) L-NAME treatment reduced ABCG2 mRNA and protein levels. "P<0.05. (B) N-PLA reduced ABCG2 expression. " $\mathrm{P}<0.05$. (C) ABCG2 was expressed to a higher level in NOS1 overexpressing cells compared with in control cells. "P<0.05. (D) The expression of ABCG2 in NOS1-overexpressing tissues was higher than in the control. Magnification, $\mathrm{x} 400$; scale bar, $20 \mu \mathrm{m}$. " $\mathrm{P}<0.05$. NOS1, nitric oxide synthase 1; ABCG2, ATP-binding cassette, subfamily G, member 2; L-NAME, ${ }^{\omega}$-nitro-L-arginine methyl ester hydrochloride; N-PLA, ${ }^{\omega}$-propyl-L-arginine hydrochloride; Con, control.
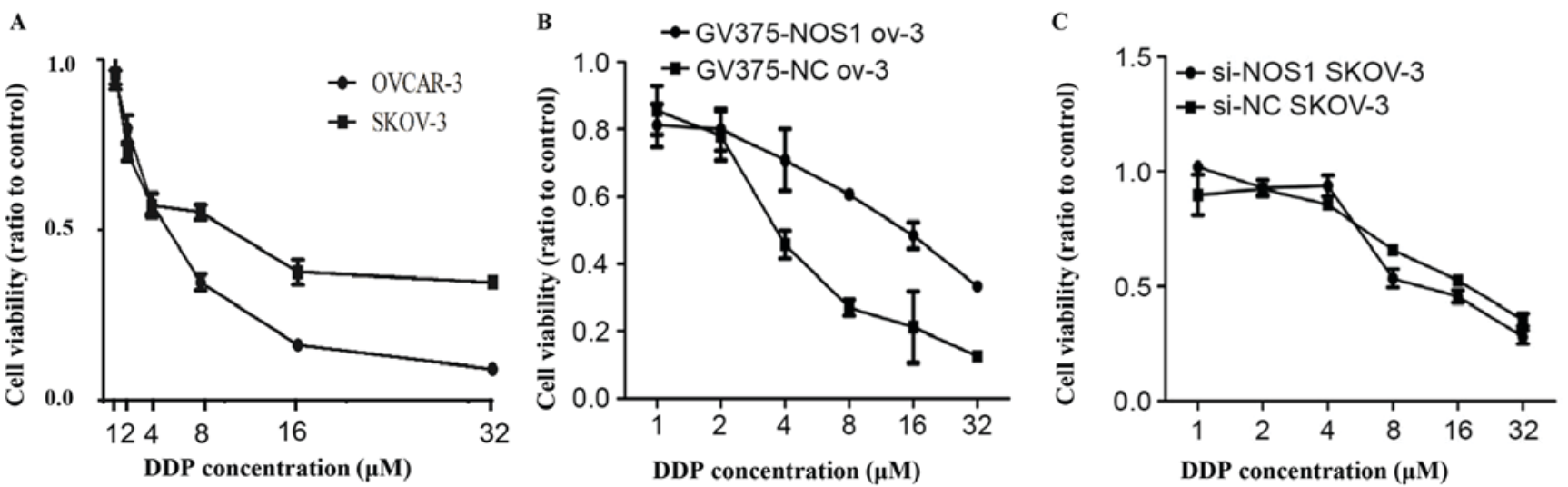

Figure 3. NOS1 contributed to DDP-resistance in ovarian cancer cell lines. (A) SKOV-3 cells exhibited greater resistance to DDP than OVCAR-3 cells. (B) OVCAR-3 cells constitutively overexpressing NOS1 were more resistant to DDP than negative controls. (C) Following transfection with si-NOS1, DDP resistance of SKOV-3 cells tended to be lower than that of negative controls. Values are presented as ratios compared with untreated cells, and are the means \pm SD of 8-10 wells. NOS1, nitric oxide synthase 1; DPP, cisplatin/cis-diamminedichloroplatinum; si, small interfering RNA; -NC, negative control.

assay to investigate whether NOS1 expression was associated with DDP resistance. Consistent with published findings that SKOV-3 is more chemoresistant than OVCAR-3 (21), the present study observed that the IC50 of SKOV-3 and OVCAR-3 cells for cisplatin was $11.3193 \pm 0.192$ and $6.5093 \pm 1.902 \mu \mathrm{M}$, respectively (Fig. 3A). Subsequently, NOS1 was overexpressed in OVCAR-3 cells and it was revealed that DDP resistance was enhanced in the cells following the overexpression, compared with controls (Fig. 3B). Meanwhile, knockdown of NOS1 by using RNA interference in SKOV-3 cells somewhat reduced the DDP resistance at higher concentrations (8-32 $\mu \mathrm{M})$ of DPP (Fig. 3C). These results indicated that NOS1 expression may partially promote DDP resistance in ovarian carcinoma.

NOS1-induced DDP resistance is reversed by $A B C G 2$ inhibition. In order to detect whether $\mathrm{ABCG} 2$ serves a role in NOS1-induced chemoresistance, the ABCG2 inhibitor verapamil was used. GV375-NOS1 ov-3 and negative control 
cells were incubated with DDP only or DDP plus verapamil, a calcium antagonist used as an ABCG2 inhibitor, for $48 \mathrm{~h}$ and the ratios of viable cells were measured via MTT assay. The cell viability of GV375-NOS1 ov-3 cells was higher $(0.841 \pm 0.038)$ than that of the negative controls $(0.641 \pm 0.021$; $\mathrm{P}<0.05)$, and this effect was significantly reversed by verapamil $(0.639 \pm 0.025 ; \mathrm{P}<0.05$; Fig. 4). These results indicated that the function of ABCG2 contributed to NOS1-related chemoresistance.

NOS1 inhibitor increases DDP-induced apoptosis and cell death. As an effect of NOS1 on DDP-resistance was observed in ovarian cancer cells, it was next investigated whether NOS1 inhibition could enhance the apoptosis and cell death induced by DDP treatment. For this, an Annexin-V/PI apoptosis assay was performed to investigate the levels of non-apoptotic cell death and apoptosis in OVCAR-3 cells following DPP exposure (Fig. 5). In this experiment, 5 treatment groups were investigated: DDP alone or with either L-NAME, N-PLA, 1400W or DETA. Fig. 5B presents the flow cytometry scatter plots of the 5 treatment groups and negative control. In all scatter plots, the $\mathrm{X}$ axis presented fluorescence intensity of FITC Annexin-V, meanwhile $\mathrm{Y}$ axis presented fluorescence intensity of PI. In each single plot, cells in the first quadrant (Q1) were FITC-/PI+. Cells in the second quadrant (Q2) were FITC+/PI+(non-apoptotic death cells). Cells in the third quadrant (Q3) were FITC+/PI-(apoptotic cells). Cells in the fourth quadrant (Q4) were FITC-/PI-(viable cells). As depicted in Fig. 5C and D, addition of N-PLA, the NOS1 selective inhibitor, significantly increased the levels of apoptosis and non-apoptotic cell death induced by DDP in OVCAR-3 cells as compared with DDP alone $(\mathrm{P}<0.05)$. By contrast, combination of DPP with the NOS2 selective inhibitor $1400 \mathrm{~W}$ appeared to somewhat decrease the DDP-induced apoptosis of cells (Fig. 5C), while the change of non-apoptotic cell death was not apparent (Fig. 5D). L-NAME, as a general inhibitor of all NOS isoforms, exhibited a mixed effect through NOS1 and 2 inhibition (Fig. 5C and D). In general, these data appear to suggest that NOS1 promoted the survival of ovarian cancer cells.

The NO donor DETA-NONOate may mimic paradoxical functions of the three types of NOS by releasing different concentrations of $\mathrm{NO}(22,23)$. In the current experiments, a low concentration of $\mathrm{NO}$ was maintained by the addition of a low dosage of DETA-NONOate $(20 \mu \mathrm{M})$ in order to simulate the effect of NOS1. It was observed that the low dose of DETA-NONOate had a mildly reducing effect on DDP-induced apoptosis, but no effect on DDP-induced non-apoptosis cell death (Fig. 5C and D). These data indicated that the low dosage of DETA-NONOate may have simulated a NOS1 effect in protecting ovarian cancer cells from DDP-mediated cytotoxicity.

To investigate whether NOS1 inhibition could improve sensitivity to DDP chemotherapy, OVCAR-3 cells were incubated with DDP only or DDP plus L-NAME or N-PLA for $48 \mathrm{~h}$, and the percentage of viable cells was assessed by MTT assay. The cells incubated with L-NAME or N-PLA exhibited enhanced sensitivity to DDP (Fig. 5A; $\mathrm{P}<0.05$ ).

Collectively, these data indicated that NOS1 had a function of reducing the rate of cell apoptosis and cell death under DDP treatment, thereby enhancing chemoresistance to DDP in ovarian cancer cells.

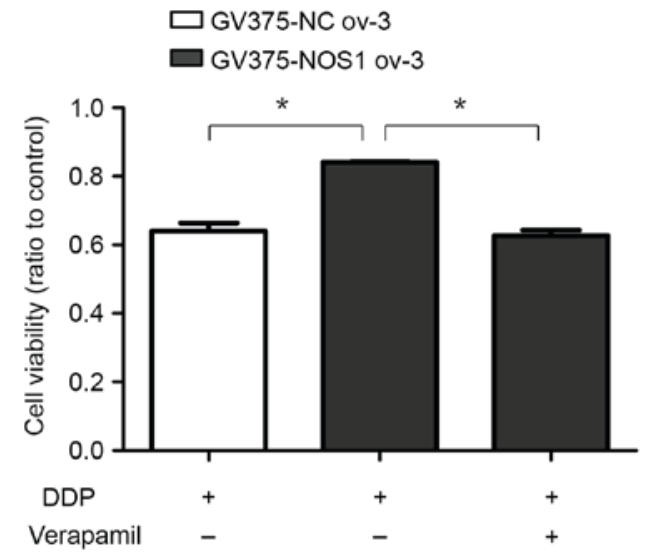

Figure 4. NOS1 induced DDP-resistance was reversed by ABCG2 inhibitor. Under DDP treatment, NOS1 overexpressing cells exhibited greater cell viability than control cells. When combined with verapamil, this effect was significantly reversed. Values are presented as ratios compared with untreated cells, and are the means \pm SD of $8-10$ wells. ${ }^{*} \mathrm{P}<0.05$. NOS1, nitric oxide synthase 1; DPP, cisplatin/cis-diamminedichloroplatinum; ABCG2, ATP-binding cassette, subfamily G, member 2; -NC, negative control.

\section{Discussion}

The impact of $\mathrm{NO}$ on malignant biological properties is complex, and NO production depends partly on the NOS isoform (6). NOS1 and NOS3 are constitutively expressed in cells, and both produce low levels of NO. In contrast, NOS2 becomes increasingly expressed in response to inflammation and produces high levels of $\mathrm{NO}$ in a $\mathrm{Ca}^{2+}$-independent manner (3). Enhanced expression of NOS isoforms has been determined in a range of cancers (24). The 3 isoforms of NOS produce endogenous NO during arginine metabolism. The protumor effects of NO occur at low concentrations, while at higher concentrations anti-tumor effects are typically induced (4). Previously, it has been reported that NOS1 could produce transient, low levels of NO, compared with the higher and more sustained concentrations of NO generated by NOS2 (25). A previous study has revealed that the 3 types of NOS were differentially regulated in chemosensitive and chemoresistant cells, and DDP resistance was associated with low NOS2 content and a high level of NOS1 in DDP-unresponsive cells (9). Thus, NOS1 appears to have a tendency to promote tumor chemoresistance. The present findings suggested that NOS1 promotes DDP chemotherapeutic resistance in ovarian cancer cell lines. By using NOS1-selective inhibitor, NOS1 was indicated to increase DDP-induced cell apoptosis and non-apoptotic cell death. To investigate the mechanism of NOS1 in promoting DDP resistance, the expression of the multidrug resistance associated gene ABCG2 was detected, and it was confirmed that suppression of $\mathrm{ABCG} 2$ function could reverse DDP-resistance induced by NOS1 expression.

However, the exact mechanism underlying the upregulation of ABCG2 expression via NOS1 remains unclear. Several transcription factors, including nuclear factor- $\kappa \mathrm{B}$ (NF-kB), HIFs and Nrf2 have been demonstrated to bind to their response elements in the promoter/enhancer region to activate the transcription of ABCG2 (26-28). Disruption of Nrf2 expression in lung cancer and prostate cancer cells 
A

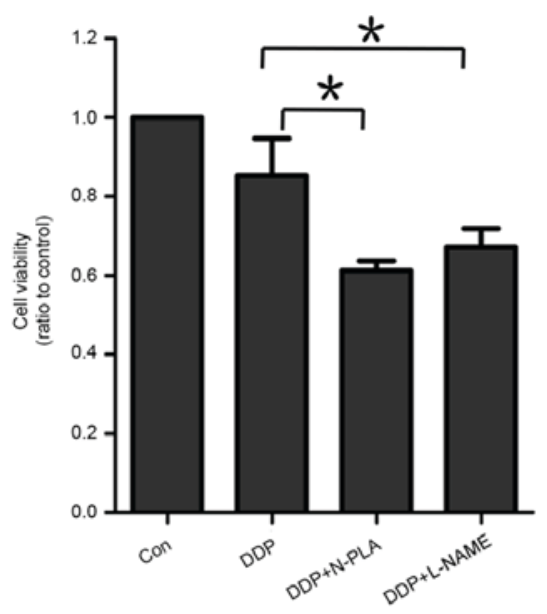

B
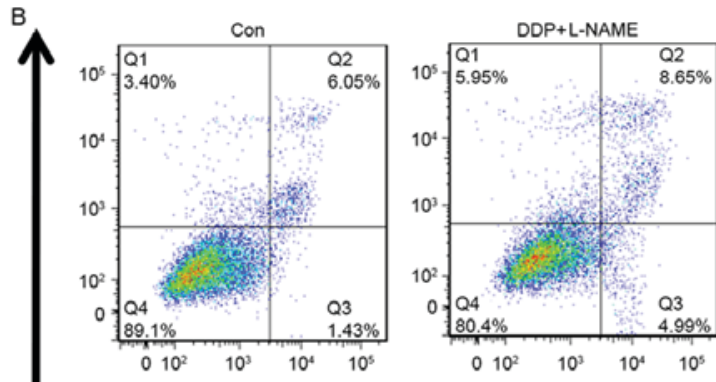

$\frac{\pi}{a}$
잉
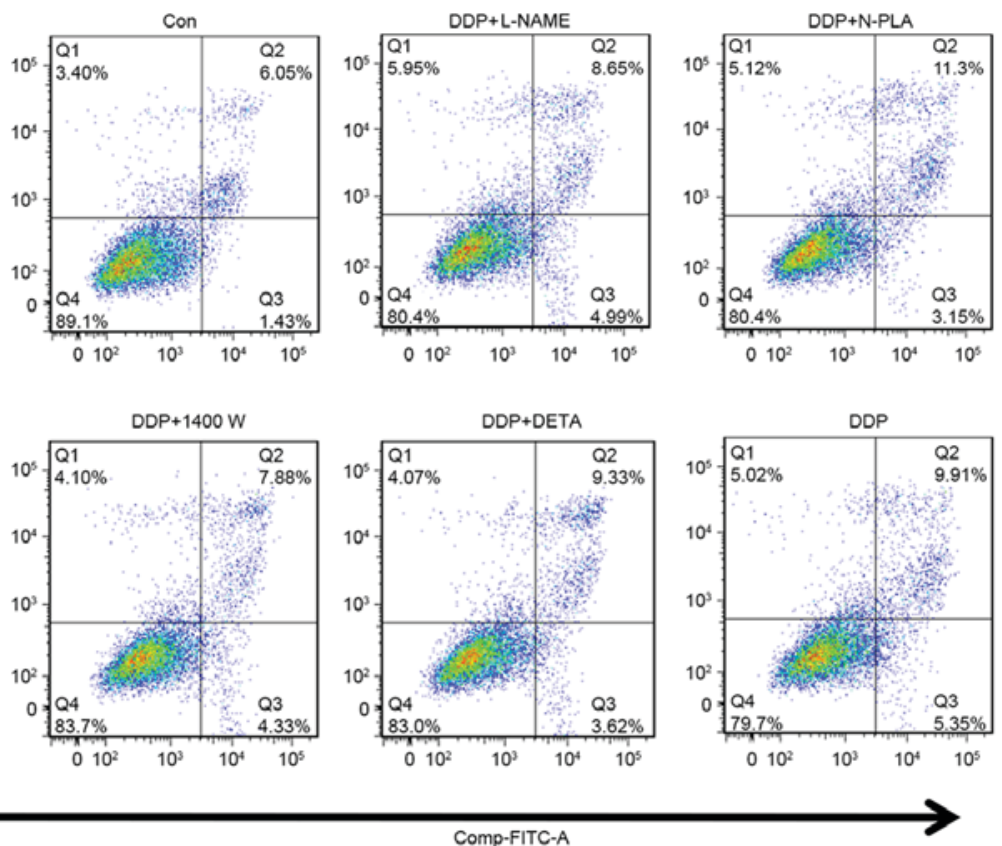

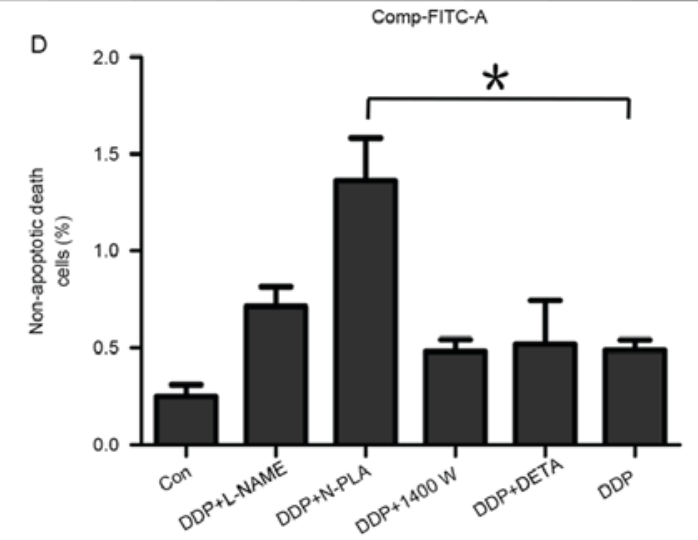

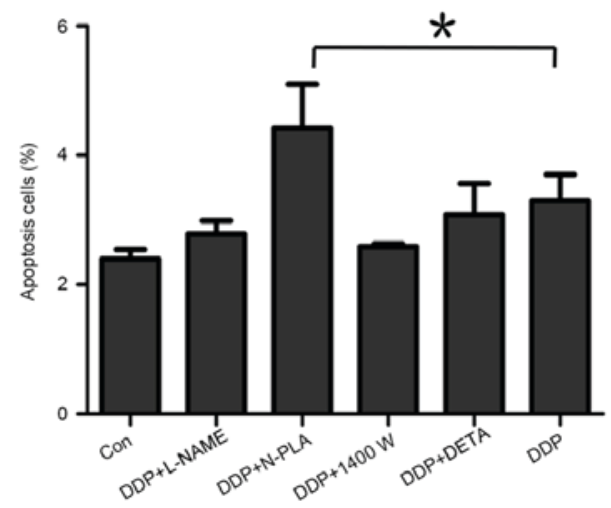

Figure 5. NOS1 enhanced the DDP-induced cell apoptosis and non-apoptotic cell death. (A) When under chemotherapeutic stress, L-NAME and N-PLA sensitized OVCAR-3 cells to DDP. (B, C and D) Apoptotic profiles of cells treated with DDP and NOS inhibitors or NO donor (DETA-NONOate). NOS1 selective inhibitor N-PLA significantly increased apoptosis and non-apoptotic cell death induced by DDP. ${ }^{*} \mathrm{P}<0.05$. NOS1, nitric oxide synthase 1; DPP, cisplatin/cis-diamminedichloroplatinum; L-NAME, $\mathrm{N}^{\omega}$-nitro-L-arginine methyl ester hydrochloride; N-PLA, $\mathrm{N}^{\omega}$-propyl-L-arginine hydrochloride; DETA,DETA-NONOate; Con, control.

by small hairpin RNA attenuated the expression of the ABCG2 transcript and protein in Nrf2-depleted cancer cells (27). Furthermore, depleted levels of ABCG2 in these Nrf2-knockdown cells caused sensitization to mitoxantrone and topotecan, two chemotherapy drugs detoxified mainly by ABCG2. In addition, it has been reported that ABCG2 expression may be activated by NF- $\mathrm{kB}$ through direct DNA binding and downregulated by wt-p53 through a decrease in NF- $\mathrm{KB}$ activity in MCF-7 cells (26). Another study has revealed a cytoprotective functional role of $\mathrm{ABCG} 2$ in response to oxidative stress, occurring downstream of HIF-2 $\alpha$. It also found a dose-dependent activation of ABCG2 expression by HIF-2 $\alpha$ (28). As mentioned, NOS1, which produces low levels of NO, may act as an environmental stimulus to activate oxidative stress-related transcription factors. Thus, it may be assumed that this is one potential mechanism by which NOS1 upregulates ABCG2 expression. This is a current area of focus and the intended subject of prospective studies by our group. Overall, it may be concluded that the NOS1-induced enhancement of DDP chemotherapeutic resistance in ovarian cancer cells is mediated by increased expression of ABCG2.

Among the greatest issues compromising the successful treatment of ovarian cancer is chemoresistance (29). It has been reported that NOS and its product NO serves roles in chemoresistance $(7,30,31)$. However, understanding of the potential mechanisms involved is still limited. The present study demonstrated that NOS1 regulated the expression level of ABCG2 and contributed to DDP-induced chemoresistance in an ABCG2-dependent manner. In summary, the current data support NOS1 as a valid tumor therapeutic target and suggest that NOS1 inhibitor used in conjunction with first-line chemotherapy may be a useful therapeutic strategy in the treatment of patients with EOC.

\section{Acknowledgements}

Not applicable. 


\section{Funding}

The present study was supported by the Fund for Technological Innovation Projects at the Department of Education of Guangdong, China (grant no. 2013KJCX0042), the Specialized Research Fund for the Doctoral Program of Higher Education by the Ministry of Education of the People's Republic of China (grant no. 20134433110009) and the Special Clinical Scientific Research Fund of Wu Jieping Medical Foundation (grant no. 320.6755.15010).

\section{Availability of data and materials}

The datasets used and/or analyzed during the current study are available from the corresponding author on reasonable request.

\section{Authors' contributions}

XL performed all experiments and $\mathrm{ZZ}$ assisted performing cells cultures and western blotting. QL, YW and XL designed the study. JT assisted in performing IHC staining. YZ assisted in RNA extraction. YLi and YLu analyzed experimental data. YW reviewed and edited the manuscript. All authors read and approved the final manuscript.

\section{Ethics approval and consent to participate}

The animal experiments were approved by the Ethics Committee of Southern Medical University (2015-FCK-002).

\section{Patient consent for publication}

Not applicable.

\section{Competing interests}

The authors declare that they have no competing interests.

\section{References}

1. Sundar S, Neal RD and Kehoe S: Diagnosis of ovarian cancer. BMJ 351: h4443, 2015.

2. Burke AJ, Sullivan FJ, Giles FJ and Glynn SA: The yin and yang of nitric oxide in cancer progression. Carcinogenesis 34 503-512, 2013.

3. Lala PK and Chakraborty C: Role of nitric oxide in carcinogenesis and tumour progression. Lancet Oncol 3: 149-156, 2001.

4. Fukumura D, Kashiwagi S and Jain RK: The role of nitric oxide in tumour progression. Nat Rev Cancer 6: 521-534, 2006.

5. Rao CV: Nitric oxide signaling in colon cancer chemoprevention. Mutat Res 555: 107-119, 2004.

6. Ridnour LA, Thomas DD, Switzer C, Flores-Santana W, Isenberg JS, Ambs S, Roberts DD and Wink DA: Molecular mechanisms for discrete nitric oxide levels in cancer. Nitric Oxide 19: 73-76, 2008.

7. Fetz V, Bier C, Habtemichael N, Schuon R, Schweitzer A, Kunkel M, Engels K, Kovacs AF, Schneider S, Mann W, et al: Inducible NO synthase confers chemoresistance in head and neck cancer by modulating survivin. Int J Cancer 124: 2033-2041, 2009

8. Matthews NE, Adams MA, Maxwell LR, Gofton TE and Graham CH: Nitric oxide-mediated regulation of chemosensitivity in cancer cells. J Natl Cancer Inst 93: 1879-1885, 2001.

9. Leung EL, Fraser M, Fiscus RR and Tsang BK: Cisplatin alters nitric oxide synthase levels in human ovarian cancer cells: Involvement in p53 regulation and cisplatin resistance. $\mathrm{Br}$ J Cancer 98: 1803-1809, 2008.
10. Robey RW, To KK, Polgar O, Dohse M, Fetsch P, Dean M and Bates SE: ABCG2: A perspective. Adv Drug Deliv Rev 61: 3-13, 2009.

11. Nakanishi T and Ross DD: Breast cancer resistance protein (BCRP/ABCG2): Its role in multidrug resistance and regulation of its gene expression. Chin J Cancer 31: 73-99, 2012.

12. Brechbuhl HM, Gould N, Kachadourian R, Riekhof WR, Voelker DR and Day BJ: Glutathione transport is a unique function of the ATP-binding cassette protein ABCG2. J Biol Chem 285: 16582-16587, 2010.

13. Valko M, Leibfritz D, Moncol J, Cronin MT, Mazur M and Telser J: Free radicals and antioxidants in normal physiological functions and human disease. Int J Biochem Cell Biol 39: 44-84, 2007.

14. Kim BR, Seo SH, Park MS, Lee SH, Kwon Y and Rho SB: sMEK1 inhibits endothelial cell proliferation by attenuating VEGFR-2-dependent-Akt/eNOS/HIF-1 $\alpha$ signaling pathways. Oncotarget 6: 31830-31843, 2015.

15. GangulaP,RavellaK,Chukkapalli S,Rivera M,SrinivasanS,Hale A, Channon K, Southerland J and Kesavalu L: Polybacterial periodontal pathogens alter vascular and gut BH4/nNOS/NRF2-Phase II enzyme expression. PLoS One 10: e0129885, 2015.

16. Castaldo SA, Freitas JR, Conchinha NV and Madureira PA: The tumorigenic roles of the cellular REDOX regulatory systems. Oxid Med Cell Longev 2016: 8413032, 2016.

17. Dasari S and Tchounwou PB: Cisplatin in cancer therapy: Molecular mechanisms of action. Eur J Pharmacol 740: 364-378, 2014.

18. Malpica A, Deavers MT, Lu K, Bodurka DC, Atkinson EN, Gershenson DM and Silva EG: Grading ovarian serous carcinoma using a two-tier system. Am J Surg Pathol 28: 496-504, 2004.

19. Livak KJ and Schmittgen TD: Analysis of relative gene expression data using real-time quantitative PCR and the 2(-Delta Delta C(T)) method. Methods 25: 402-408, 2001.

20. Baig MS, Zaichick SV, Mao M, de Abreu AL, Bakhshi FR, Hart PC, Saqib U, Deng J, Chatterjee S, Block ML, et al: NOS1-derived nitric oxide promotes NF-kB transcriptional activity through inhibition of suppressor of cytokine signaling-1. J Exp Med 212: 1725-1738, 2015.

21. Penzvalto Z, Lanczky A, Lénárt J, Meggyesházi N, Krenács T, Szoboszlai N, Denkert C, Pete I and Győrffy B: MEK1 is associated with carboplatin resistance and is a prognostic biomarker in epithelial ovarian cancer. BMC Cancer 14: 837, 2014.

22. Thomas DD, Ridnour LA, Isenberg JS, Flores-Santana W, Switzer CH, Donzelli S, Hussain P, Vecoli C, Paolocci N, Ambs S, et al: The chemical biology of nitric oxide: Implications in cellular signaling. Free Radic Biol Med 45: 18-31, 2008.

23. Xu W, Liu LZ, Loizidou M, Ahmed M and Charles IG: The role of nitric oxide in cancer. Cell Res 12: 311-320, 2002.

24. Vannini F, Kashfi K and Nath N: The dual role of iNOS in cancer. Redox Biol 6: 334-343, 2015.

25. Nathan $C$ and Xie QW: Nitric oxide synthases: Roles, tolls, and controls. Cell 78: 915-918, 1994.

26. Wang X, Wu X, Wang C, Zhang W, Ouyang Y, Yu Y and He Z: Transcriptional suppression of breast cancer resistance protein (BCRP) by wild-type p53 through the NF-kappaB pathway in MCF-7 cells. FEBS Lett 584: 3392-3397, 2010.

27. Singh A, Wu H, Zhang P, Happel $\mathrm{C}$, Ma J and Biswal S: Expression of ABCG2 (BCRP) is regulated by Nrf2 in cancer cells that confers side population and chemoresistance phenotype. Mol Cancer Ther 9: 2365-2376, 2010.

28. Martin CM, Ferdous A, Gallardo T, Humphries C, Sadek H, Caprioli A, Garcia JA, Szweda LI, Garry MG and Garry DJ: Hypoxia-inducible factor-2alpha transactivates Abcg2 and promotes cytoprotection in cardiac side population cells. Circ Res 102: 1075-1081, 2008.

29. Bast RC Jr, Hennessy B and Mills GB: The biology of ovarian cancer: New opportunities for translation. Nat Rev Cancer 9: 415-428, 2009.

30. Esteban FJ, Horcajadas A, El-Rubaidi O, Luque-Barona R, Ibáñez G, Garcia-Carriazo A, Segovia M and del Moral-Leal ML: Nitric oxide in malignant astrocytes. Rev Neurol 40: 437-440, 2005 (In Spanish).

31. Yang DI, Yin JH, Mishra S, Mishra R and Hsu CY: NO-mediated chemoresistance in C6 glioma cells. Ann N Y Acad Sci 962: 8-17, 2002.

This work is licensed under a Creative Commons Attribution-NonCommercial-NoDerivatives 4.0 International (CC BY-NC-ND 4.0) License. 